'Unidad de Endocrinología, Departamento de Medicina Interna, Clínica Alemana de Santiago, Santiago, Chile. ${ }^{2}$ Facultad de Medicina Clínica Alemana, Universidad del Desarrollo, Santiago, Chile. ${ }^{3}$ Sección Endocrinología, Hospital de Salvador, Santiago, Chile.

${ }^{4}$ Facultad de Medicina, Campus Oriente, Universidad de Chile, Santiago, Chile. ${ }^{5}$ Clínica Universidad de los Andes, Santiago, Chile.

${ }^{6}$ Fundación Arturo López Pérez, Santiago, Chile.

${ }^{7}$ Departamento de Endocrinología, Facultad de Medicina, Pontificia Universidad Católica de Chile, Santiago, Chile.

${ }^{8}$ Departamento Medicina Interna, Centro EPICYN, Facultad de Medicina Universidad de la Frontera, Temuco, Chile.

${ }^{9}$ Hospital Clínico Universidad de Chile Santiago, Chile.

${ }^{10}$ Cirugía Cabeza y Cuello, Hospital Clínico Universidad de Chile, Santiago, Chile. 'Departamento de Radiología, Pontificia

Universidad Católica de Chile, Santiago, Chile.

${ }^{12}$ Imagenología, Clínica Santa María, Santiago, Chile.

${ }^{13}$ Departamento de Cirugía Oncológica, Pontificia Universidad Católica de Chile Santiago, Chile

${ }^{14}$ División de Pediatría, Escuela de Medicina Pontificia Universidad Católica de Chile Santiago, Chile.

${ }^{15}$ Endocrinología, Hospital San BorjaArriarán, Santiago, Chile.

${ }^{16}$ Clínica Las Condes, Santiago, Chile.

${ }^{17}$ Medicina Nuclear, Clínica Las Condes, Santiago, Chile.

${ }^{18} \mathrm{Centro}$ de Diagnóstico Plaza Italia Santiago, Chile

${ }^{19}$ Unidad de Cirugía de Cabeza y Cuello, Departamento de Cirugía, Clínica Alemana de Santiago, Santiago, Chile.

${ }^{20} \mathrm{Hospital}$ San Juan de Dios, Santiago, Chile

${ }^{21}$ Pontificia Universidad Católica de Chile, Santiago, Chile.

${ }^{22}$ Hospital Clínico San Borja-Arriarán Santiago, Chile

${ }^{23}$ Facultad de Medicina, Campus Central, Universidad de Chile, Santiago, Chile.

${ }^{24}$ Departamento de Medicina Interna, Universidad de La Frontera, Temuco, Chile.

${ }^{25}$ Hospital Padre Hurtado, Santiago, Chile.

${ }^{26}$ Departamento de Anatomía Patológica,

Clínica Alemana de Santiago, Santiago, Chile

${ }^{27}$ Departamento de Anatomía Patológica, Pontificia Universidad Católica de Chile, Santiago, Chile

${ }^{28}$ Departamento de Medicina Interna, Facultad de Medicina Universidad de Concepción, Concepción, Chile.

${ }^{29}$ Hospital Militar, Santiago, Chile.

Los autores indicados con * contribuyeron de igual forma con el trabajo.

Correspondencia a: Dr. Hernán Tala

Av Vitacura 5951, Vitacura, Región Metropolitana, Santiago, Chile. htala@alemana.cl

\section{Estudio y manejo de nódulos tiroideos por médicos no especialistas. Consenso SOCHED}

\author{
HERNÁN TALA ${ }^{1,2, *}$, RENÉ E. DÍAZ $3,4,5,6,{ }^{3,}$, \\ JOSÉ MIGUEL DOMÍNGUEZ RUIZ-TAGLE7, \\ JORGE SAPUNAR ZENTENO ${ }^{8}$, PEDRO PINEDA 9 , \\ PATRICIA ARROYO ALBALA ${ }^{9}$, MARCELA BARBERÁN", \\ PATRICIO CABANÉ ${ }^{5,10}$, FRANCISCO CRUZ OLIVOS ${ }^{11}$, \\ PATRICIO GAC E. ${ }^{5,10}$, ANDREA GLASINOVIC PIZARRO ${ }^{12}$, \\ HERNÁN E. GONZÁLEZ ${ }^{13}$, FRANCISCA GROB ${ }^{14}$, \\ MARIA SOLEDAD HIDALGO VALLE ${ }^{15,16}$, RODRIGO JAIMOVICH ${ }^{17}$, \\ ALEJANDRA LANAS ${ }^{9}$, CLAUDIO LIBERMAN 9 , \\ MAITE LOBO GUIÑEZ ${ }^{18}$, ARTURO MADRID ${ }^{19,2}$, \\ MARCELA MORENO SEGUEL ${ }^{5,20}$, LORENA MOSSO ${ }^{21}$, \\ FERNANDO MUNIZAGA C. ${ }^{22,23}$, EUGENIA ORTIZ PARADA ${ }^{24}$, \\ FERNANDO OSORIO GONNET ${ }^{1,25}$, JEANNIE SLATER MORALES ${ }^{2,26}$, \\ ANTONIETA SOLAR ${ }^{27}$, CARLOS STEHR ${ }^{28}$, \\ FÉLIX VÁSQUEZ RODRÍGUEZ ${ }^{20}$, JESÚS VÉLIZ ${ }^{3,4,6}$, \\ ROBERTO VILLASECA NAJARIAN ${ }^{29}$, NELSON WOHLLK ${ }^{3,4}$
}

\section{Study and management of thyroid nodes by non specialist physicians: SOCHED consensus}

The thyroid nodule is a frequent cause of primary care consultation. The prevalence of a palpable thyroid nodule is approximately $4-7 \%$, increasing up to $67 \%$ by the incidental detection of nodules on ultrasound. The vast majority are benign and asymptomatic, staying stable over time. The clinical importance of studying a thyroid nodule is to exclude thyroid cancer, which occurs in 5 to $10 \%$ of the nodules. The Board of SOCHED (Chilean Society of Endocrinology and Diabetes) asked the Thyroid Study Group to develop a consensus regarding the diagnostic management of the thyroid nodule in Chile, aimed at non-specialist physicians and adapted to the national reality. To this end, a multidisciplinary group of 31 experts was established among university academics, active researchers with publications on the subject and prominent members of scientific societies of endocrinology, head and neck surgery, pathology and radiology. A total of 14 questions were developed with key aspects for the diagnosis and subsequent referral of patients with thyroid nodules, which were addressed by the participants. In those areas where the evidence was insufficient or the national reality had to be considered, the consensus opinion of the experts was used through the Delphi methodology. The consensus was approved by the SOCHED board for publication.

(Rev Med Chile 2017; 145: 1028-1037)

Key words: Consensus; Practice Guideline; Thyroid Gland; Thyroid Nodule. 
L os nódulos tiroideos son un problema clínico frecuente. Los estudios epidemiológicos han mostrado que la prevalencia de un nódulo tiroideo palpable es de aproximadamente $4-7 \%$ en la población general ${ }^{1,2}$.

Sin embargo, los avances en técnicas de imágenes y la amplia disponibilidad de éstos han aumentado la pesquisa de nódulos tiroideos. Actualmente, se considera que 19 a $67 \%$ de la población presenta nódulos detectables por ecografía, siendo más frecuente en los adultos mayores, llegando a $50-70 \%$ en personas mayores de 60 años ${ }^{1-3}$.

La gran mayoría de los nódulos tiroideos son benignos y asintomáticos y se mantienen estables en el tiempo, por lo que no es recomendable hacer pesquisa de nódulos tiroideos en la población general, ni estudiar histológicamente a todos los pacientes con nódulos tiroideos. La importancia clínica de estudiar un nódulo tiroideo radica en la necesidad de excluir el cáncer de tiroides, lo cual ocurre en 5 a 10\% $0^{4,5}$, dependiendo de la edad, género, exposición a la radiación en la infancia, historia familiar y otros factores. Por esta razón, se recomienda elaborar un plan de pesquisa y estudio de nódulos tiroideos como una forma de establecer conductas uniformes, evitar procedimientos innecesarios y racionalizar los recursos. La Sociedad Chilena de Endocrinología y Diabetes (SOCHED), ha convocado a un gran número de expertos nacionales, endocrinólogos, radiólogos, anátomo-patólogos, cirujanos y médicos nucleares con el fin de redactar un documento de consenso para el estudio y manejo de los nódulos tiroideos adaptado a la realidad nacional y que esperamos sea de importante ayuda principalmente para los médicos no especialistas que se enfrentan con esta patología frecuentemente en su quehacer diario.

\section{Metodología}

El Directorio de la SOCHED solicitó al Grupo de Estudio de Tiroides el desarrollo de un pronunciamiento respecto al manejo diagnóstico del nódulo tiroideo en Chile, orientado a médicos no especialistas. Para ello, se constituyó un comité de coordinación integrado por 4 miembros del Grupo de Estudios de Tiroides, que elaboró 14 preguntas que abordaban aspectos claves para el diagnóstico y posterior derivación de pacientes con nódulo tiroideo. Fueron seleccionados 31 expertos entre académicos universitarios, investigadores activos con publicaciones en el tema y miembros destacados de sociedades científicas de endocrinología, cirugía de cabeza y cuello, anatomía patológica y radiología.

El pronunciamiento se fundamentó en la evidencia obtenida mediante una revisión exhaustiva de la literatura. En aquellos aspectos en que la evidencia fue insuficiente o había que considerar la realidad nacional se recurrió a la opinión consensuada de los expertos. El consenso se realizó por metodología Delphi, para lo cual las preguntas fueron convertidas en afirmaciones y enviadas por correo electrónico a los expertos junto con una escala visual de Likert con 5 diferentes grados de acuerdo (1. Desacuerdo total; 2. Desacuerdo parcial; 3. Incertidumbre; 4. Acuerdo parcial y 5. Acuerdo total). Se consideró "acuerdo" con la afirmación si al menos $80 \%$ de las respuestas se situaban en las categorías 4 o 5 y "desacuerdo" si una proporción similar de respuestas se situaba en las categorías 1 o 2. Si la afirmación no logra consenso entre los expertos en primera ronda, el proceso se repite informándoles el resultado y continuando hasta lograr acuerdo/desacuerdo o que la proporción de respuestas no experimente variación. En esta última situación se concluye que la pregunta que origina la afirmación no tiene respuesta ${ }^{6}$.

De acuerdo a la metodología GRADE utilizamos los términos recomendamos o recomendamos en contra y el número 1 cuando la evidencia disponible es de buena calidad o cuando existió acuerdo absoluto entre los expertos. Cuando la evidencia disponible es de baja calidad o el acuerdo entre expertos fue parcial utilizamos los términos sugerimos o sugerimos en contra y el número 2 .

Finalmente, el consenso fue redactado y enviado al directorio de SOCHED para su aprobación.

\section{1. ¿A quién y cómo se debe realizar tamizaje de nódulo tiroideo?}

No se recomienda el tamizaje a la población general. Hay estudios en otros países que demuestran que al realizar ecotomografía de tiroides en la población general, como método para detectar cáncer en forma temprana, aumenta en forma significativa el diagnóstico sin impacto en la sobrevida.

Se recomienda realizar tamizado con ecotomografía tiroidea en aquellas poblaciones en las 
cuales hay un mayor riesgo de cáncer de tiroides:

- Paciente con factores de alto riesgo para cáncer de tiroides:

- Antecedente de exposición a radiación cervical antes de los 15-18 años de edad?

- Antecedente de síndrome genético asociado a alta probabilidad de cáncer de tiroides (Anexo 1):

- Neoplasia Endocrina Múltiple tipo 2 (excepto familiares cuyo estudio genético no muestre la mutación $)^{8}$.

- Poliposis colónica familiar?.

- Complejo de Carney ${ }^{10}$.

- Enfermedad de Cowden ${ }^{11}$.

- Síndrome de Werner ${ }^{12}$.

- Se recomienda considerar en pacientes con antecedente de cáncer diferenciado de tiroides en 2 o más familiares de primer grado (Recomendación, GRADE 1).

- Se sugiere no realizar ecografía tiroidea de rutina en pacientes con tiroiditis de Hashimoto, salvo en pacientes con bocio o palpación anormal (Sugerencia, GRADE 2).

- Se recomienda no realizar tamizaje en sujetos sin los factores de riesgo arriba mencionados (Recomendación, GRADE 1).

\section{2. ¿Cuáles son las características clínicas a considerar en pacientes que consultan por un nódulo tiroideo?}

\section{Antecedentes personales y familiares}

El antecedente de radiación cervical en adolescencia o niñez, historia familiar de cáncer de tiroides y antecedentes personales o familiares de síndromes clínicos asociados a mayor riesgo de cáncer de tiroides (Anexo 1), son factores que pueden aumentar el riesgo de cáncer.

\section{Características clínicas}

Clínicamente, una masa cervical de crecimiento rápido o alteraciones persistentes en la voz, deben tomarse en cuenta para considerar un nódulo como sospechoso.

Un nódulo de crecimiento rápido y doloroso suele deberse a hemorragia intranodular, en cambio el crecimiento indoloro de un nódulo en pocas semanas, más aún si está asociado a linfadenopatía, debiera considerarse como un nódulo sospechoso y dado su comportamiento agresivo derivarse en forma prioritaria (urgente).
El compromiso infiltrativo del nervio laríngeo recurrente puede manifestarse por disfonía o voz bitonal. Igualmente, es importante señalar que debe efectuarse una anamnesis cuidadosa para descartar si los síntomas son atribuibles a él o los nódulos, considerando el tamaño de éstos, la presencia de síntomas de tubo digestivo superior (pirosis, regurgitación) y el estado emocional del paciente, dado que uno de los diagnósticos diferenciales más frecuentes son el reflujo gastroesofágico y el globus faríngeo. En general, debe tenerse en cuenta que, en el caso de nódulos no palpables, es improbable que tengan síntomas locales como los mencionados.

\section{3. ¿Cuáles son los exámenes de laboratorio necesarios para evaluar un paciente con nódulo tiroideo?}

Siempre se debe medir TSH. Si está bajo el rango de normalidad, debiera realizarse un cintigrama tiroideo si el nódulo tiroideo es $>$ de $1 \mathrm{~cm}$. Si está elevada debería procederse a un estudio mayor con T4 libre y anticuerpos anti tiroideos.

Con respecto a la medición de calcitonina plasmática, se recomienda no medirla en forma rutinaria y limitarla a casos con antecedentes familiares de cáncer medular de tiroides, antecedentes personal o familiar de feocromocitoma o hiperparatiroidismo primario o PAAF (punción aspirativa con aguja fina) informada como neoplasia folicular, especialmente neoplasia de Hürthle. Si la calcitonina sérica es $>50-100$ pg/mL el diagnóstico de cáncer medular es muy probable. Valores intermedios pueden ser repetidos con estimulación con calcio ${ }^{13}$.

\section{4. ¿Qué estudio imagenológico se debe realizar en un paciente con nódulo tiroideo?}

Se debe realizar una ecotomografía de tiroides en todo paciente en el cual clínicamente se detecta o se sospecha la presencia de un nódulo tiroideo, $y$ en aquellos en los cuales se diagnostica incidentalmente mediante otro examen de imágenes (ecotomografía doppler carotidea, tomografía axial computarizada, resonancia nuclear magnética o 18-Fluor-desoxi-glucosa- Tomografía por emisión de positrones (FDG-PET).

\section{4a. ¿Cuáles son los requisitos que debe tener el informe de la ecotomografía?}

Descripción general de la glándula tiroides: Indicar presencia o ausencia parcial o total de la 


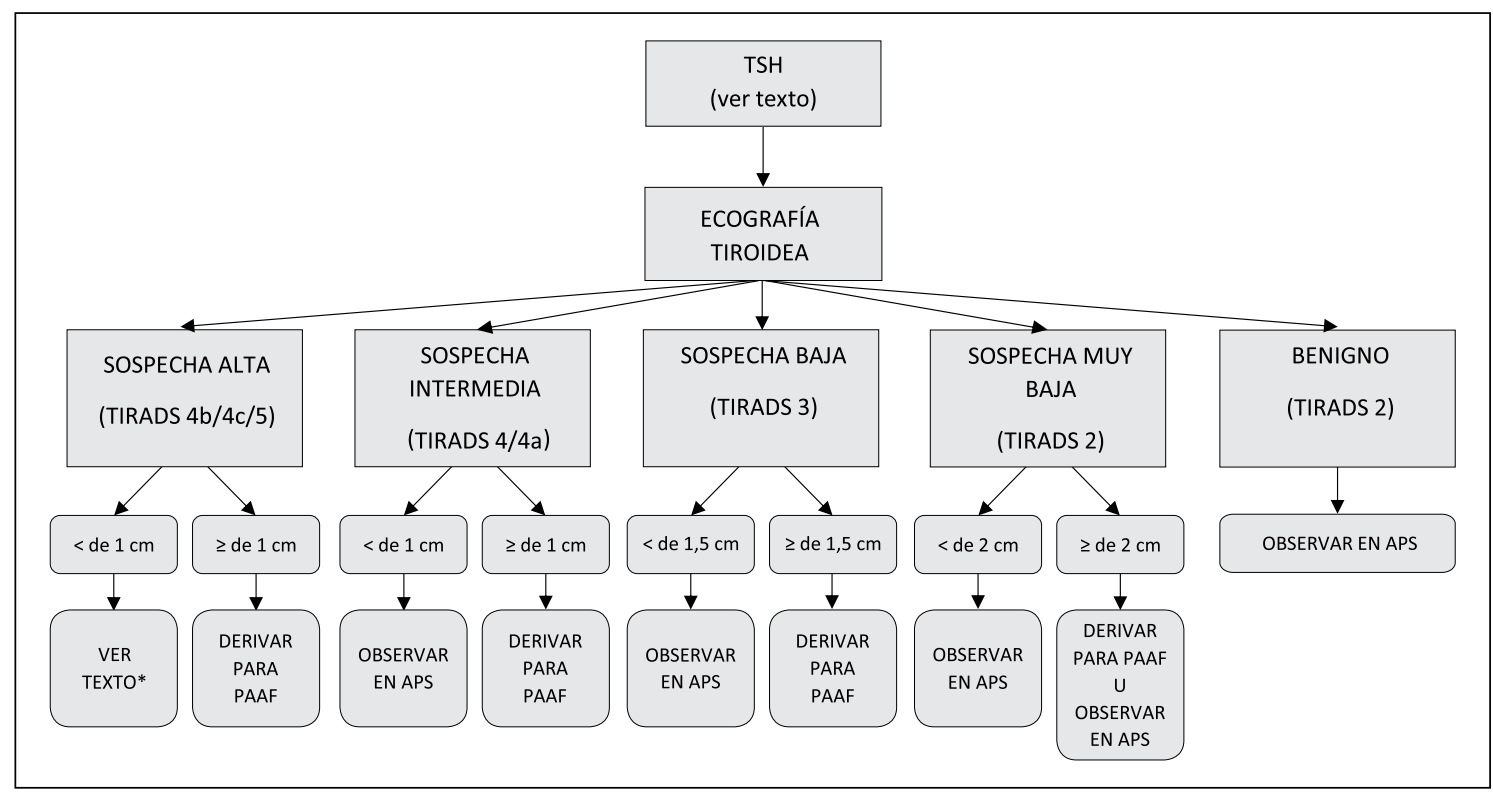

Figura 1. Diagrama de flujo estudio y derivación de paciente con nódulo tiroideo. *Derivar a especialista nódulo tiroideo $<$ $1 \mathrm{~cm}$ con criterios ecográficos de alta sospecha, asociado a adenopatías sospechosas, proximidad a tráquea o nervio laríngeo recurrente o sospecha de extensión extratiroidea; en estos casos el especialista asumirá la decisión de estudio con punción o seguimiento. En caso de no tener estas características pueden ser controlados en atención primaria o bien derivados a endocrinología. PAAF: Punción aspirativa con aguja fina; APS: Atención primaria de salud.

glándula, las dimensiones en los tres ejes: longitudinal, anteroposterior y transversal de los lóbulos, incluido el lóbulo piramidal, y diámetro anteroposterior del istmo. Se debe describir sus contornos, características del parénquima (ecogenicidad y ecoestructura) y vascularización. Evaluar presencia de quiste tirogloso.

Descripción de nódulos: ubicación, tamaño, contornos, ecoestructura, ecogenicidad y vascularización, indicar la presencia de micro y macrocalcificaciones. En bocio multinodular los otros nódulos no dominantes se deben informar separadamente, a no ser que tengan características similares.

Descripción de los linfonodos: toda ecografía tiroidea debe incluir descripción de los linfonodos del compartimento central y laterocervical que sean clínicamente significativos.

Conclusiones: El informante debe describir y concluir si el nódulo es sospechoso o no. En lo posible, se debe describir el grado de sospecha según la clasificación de la American Thyroid Association publicada el año 2015 o bien la categoría TIRADS en caso que tenga entrenamiento en dicha clasi- ficación (Anexo 2). En el informe ecográfico no debiera sugerirse la realización de biopsia u otro estudio complementario. Es el clínico quien decidirá la necesidad de estudios complementarios, incluyendo la PAAF. Lo anterior, principalmente en nódulos $<$ a $1 \mathrm{~cm}$, independiente del riesgo ecográfico de malignidad.

\section{4b. ¿Cuándo realizar Cintigrama tiroideo? ¿Tiene utilidad el 18-FDG PET CT?}

Deberá pedirse cintigrama de tiroides con $99 \mathrm{Tc}$ (tecnecio) cada vez que un paciente portador de nódulo tiroideo $>$ de $1 \mathrm{~cm}$ tenga una TSH bajo el rango normal. Si el nódulo es funcionante, el riesgo de malignidad es muy bajo y por lo tanto no requiere de PAAF salvo que ecográficamente sea de alta sospecha de malignidad.

No se ha demostrado que el uso de 18-FDG PET CT tenga alguna utilidad diagnóstica en el nódulo tiroideo. Sin embargo, aquellos nódulos que son detectados incidentalmente mediante este método tienen mayor riesgo de malignidad $(\approx 30 \%)$, por lo que deben ser estudiados dirigidamente ${ }^{14}$. 


\section{5. ¿Cuáles son las indicaciones de PAAF de un nódulo tiroideo?}

La indicación de PAAF y de derivación desde atención primaria de salud (APS) depende principalmente de las características clínicas y ultrasonográficas, teniendo en cuenta también los antecedentes personales y familiares.

En el año 2015 la American Thyroid Association (ATA) publicó sus recomendaciones para PAAF de nódulos, basadas en el riesgo de malignidad definido por las características ecográficas. Estas guías definen distintos patrones ecográficos y los correlacionan con riesgo de malignidad ${ }^{15}$.

Las distintas categorías definidas por estas guías se pueden homologar a las categorías TI-
RADS, utilizadas por algunos centros de nuestro país. La confiabilidad de la clasificación TIRADS depende de la experiencia del radiólogo ${ }^{16,17}$ (Anexo 2). Por ello, es importante que la ecografía sea efectuada en centros con experiencia (de lo contrario se sugiere solo describir las características ecográficas). En el Anexo 2 se discute en detalle esta clasificación (Recomendación, GRADE 1).

En la Tabla 1 se detallan las características ecográficas de cada categoría según la clasificación ATA 2015. Con el intento de simplificar el análisis según el reporte que se utilice, en la misma tabla describimos la categoría TIRADS asociada, y la indicación de PAAF según la categoría de riesgo (Recomendación, GRADE 1).

Tabla 1. Resumen riesgo de malignidad de nódulo tiroideo e indicación de PAAF

\begin{tabular}{|c|c|c|}
\hline Riesgo de malignidad & Características ecográficas & Indicación de PAAF \\
\hline $\begin{array}{l}\text { Alta sospecha o } \\
\text { TIRADS } 4 \mathrm{~b} / 4 \mathrm{c} / 5 \\
\text { ( } 70-90 \% \text { de riesgo de } \\
\text { malignidad) }\end{array}$ & $\begin{array}{l}\text { Nódulo sólido hipoecogénico (o el componente } \\
\text { sólido hipoecogénico de un nódulo mixto) con } \\
\text { una o más de las siguientes características: } \\
\text { - Márgenes irregulares } \\
\text { - Microcalcificaciones } \\
\text { - Más alto que ancho } \\
\text { - Calcificaciones periféricas no continuas con } \\
\text { permeación de tejidos blandos entre las calci- } \\
\text { - Excaciones } \\
\text { - Linfonsión extratiroidea }\end{array}$ & $\begin{array}{l}\text { Cuando sean } \geq \text { de } 1 \mathrm{~cm} \\
\text { Entre } 5 \text { y } 10 \mathrm{~mm} \text {, evaluar caso a caso } \\
\text { necesidad de PAAF } \\
<5 \mathrm{~mm} \text {, no puncionar, salvo presen- } \\
\text { cia de adenopatías con aspecto de } \\
\text { metástasis o extensión extratiroidea, } \\
\text { sospecha de compromiso traqueal o } \\
\text { de nervio laríngeo recurrente }\end{array}$ \\
\hline $\begin{array}{l}\text { Sospecha intermedia o } \\
\text { TIRADS } 4 / 4 \text { a } \\
\text { (10-20\% riesgo de malignidad) }\end{array}$ & $\begin{array}{l}\text { Nódulo sólido hipoecogénicos con márgenes } \\
\text { bien definidos (SIN microcalcificaciones, exten- } \\
\text { sión extratiroidea o más alto que ancho) }\end{array}$ & Cuando sean $\geq$ de $1 \mathrm{~cm}$ \\
\hline $\begin{array}{l}\text { Baja sospecha o TIRADS } 3 \\
\text { (5-10\% riesgo de malignidad) }\end{array}$ & $\begin{array}{l}\text { Nódulo sólido isoecogénico o hiperecogénico o } \\
\text { parcialmente quísticos con áreas sólidas } \\
\text { (SIN microcalcificaciones, margen irregular, más } \\
\text { alto que ancho o extensión extratiroidea) }\end{array}$ & Cuando sean $\geq 1,5 \mathrm{~cm}$ \\
\hline $\begin{array}{l}\text { Muy baja sospecha o TIRADS } 2 \\
(<3 \% \text { de riesgo de malignidad) }\end{array}$ & $\begin{array}{l}\text { Espongiformes (aspecto de múltiples microquis- } \\
\text { tes que ocupan }>50 \% \text { del volumen nodular) o } \\
\text { parcialmente quístico }\end{array}$ & $\begin{array}{l}\text { Cuando sean } \geq 2 \mathrm{~cm} \text { o también se } \\
\text { podría solo observar }\end{array}$ \\
\hline $\begin{array}{l}\text { Benigna o TIRADS } 2 \\
(<1 \% \text { de riesgo de malignidad })\end{array}$ & Nódulo quístico puro (sin componente sólido) & No requiere PAAF \\
\hline Situación especial & $\begin{array}{l}\text { Presencia de: } \\
\text { - Adenopatías sospechosas } \\
\text { - Extensión extratiroidea } \\
\text { - Compromiso Traqueal } \\
\text { - Compromiso de Nervio laríngeo recurrente }\end{array}$ & $\begin{array}{l}\text { PAAF siempre, independiente de } \\
\text { tamaño }\end{array}$ \\
\hline & $\begin{array}{l}\text { Antecedentes: } \\
\text { - Síndromes familiares } \\
\text { - Exposición a radiación antes de los } 18 \text { años }\end{array}$ & $\begin{array}{l}\text { Se debe tener en consideración en la } \\
\text { toma de decisiones }\end{array}$ \\
\hline
\end{tabular}

Es importante considerar que las características del nódulo se pueden modificar con la PAAF. 


\section{6. ¿Cuál es la conducta en nódulos sospechosos menores a $1 \mathrm{~cm}$ ?}

Aun cuando el patrón ecográfico sea altamente sospechoso, es muy discutible el beneficio clínico de la PAAF y eventual posterior cirugía de nódulos menores de $1 \mathrm{~cm}$. La prevalencia de microcarcinoma papilar de tiroides es alta en la población general, y la probabilidad de progresar a tumores de mayor tamaño o de generar metástasis ganglionares es baja en estudios donde se ha ofrecido observación en este grupo de pacientes ${ }^{18,19}$.

Es por ello que en nódulos $<5 \mathrm{~mm}$, aun siendo de alto riesgo ecográfico, se recomienda observar (salvo los raros casos que presenten ganglios con sospecha de metástasis, extensión extratiroidea, compromiso traqueal o de nervio laríngeo recurrente) (Recomendación, GRADE 1).

En el caso de nódulo sospechoso entre 5-10 $\mathrm{mm}$, se deben considerar una serie de factores para evaluar la necesidad y posible beneficio de PAAF e intervención en caso de ser un microcarcinoma, como ubicación del nódulo (adyacente a tráquea o a nervio laríngeo recurrente), sospecha de extensión extratiroidea, adenopatías sospechosas, edad del paciente y antecedentes familiares, entre otras $^{18-21}$. Se debe considerar derivar al especialista nódulos altamente sospechosos entre 5 y $10 \mathrm{~mm}$. Por otro lado, todo nódulo menor a $1 \mathrm{~cm}$ con sospecha de extensión extratiroidea, en contacto a la tráquea o con nervio laríngeo recurrente o con metástasis ganglionares debe ser derivado para PAAF (Recomendación, GRADE 1).

\section{7. ¿Cómo es la técnica de PAAF por aguja fina?}

En el estudio y manejo del nódulo tiroideo se recomienda ampliamente la PAAF, con aguja 19 o $21 \mathrm{G}$, utilizando las técnicas de block celular, coágulo y citología, según sea la experiencia de cada centro.

Para mejorar el rendimiento de la PAAF, todos los nódulos que requieran PAAF (incluyendo los palpables) deben ser puncionados bajo guía de ecografía empleando transductores adecuados (5-12 o 5-17 MHz).

\section{8. ¿Cómo debe ser analizada la muestra obteni- da por PAAF?}

Debe ser hecho por un patólogo con experiencia en PAAF de tiroides o dedicado a la patología tiroidea y debe ser reportada usando las categorías diagnósticas del Sistema Bethesda (The Bethesda
System for Reporting Thyroid Cytopathology $)^{22,23}$. En la Tabla 2 se describe el porcentaje de nódulos cuyo diagnóstico corresponde a cada una de las categorías Bethesda y la probabilidad de malignidad según cada una de ellas (de acuerdo a lo reportado por la publicación original de Bethesda ${ }^{22}$.

Si una misma muestra fue procesada de distintas formas (frotis, coágulo y/o block celular), pero fueron obtenidas del mismo nódulo, el diagnóstico debe ser único tomando en cuenta todas las muestras obtenidas, aunque hayan sido procesadas de distintas formas.

Con respecto al control de calidad, es importante que cada laboratorio de anatomía patológica analice sus propias estadísticas, evaluando el porcentaje de nódulos cuyas PAAF corresponde a cada diagnóstico en particular, y a su vez, la correlación citológica/histológica en los casos que van a cirugía 22,23 .

\section{9. ¿Cuáles son las indicaciones de derivación de pacientes con nódulo tiroideo desde Atención Primaria?}

Dado que la mayoría de los nódulos tiroideos son benignos, debe efectuarse una correcta interpretación de la clínica y de la ecografía tiroidea ante el hallazgo de uno o múltiples nódulos.

\subsection{Casos que requieren evaluación en policlíni-} co de endocrinología:

a) Nódulo tiroideo con criterios ecográficos de sospecha de malignidad $\mathrm{y} \geq 1 \mathrm{~cm}$ con indicación de PAAF (ver indicaciones de PAAF de nódulo tiroideo).

Tabla 2. Frecuencia y probabilidad de malignidad según categoría de Bethesda (de acuerdo a lo reportado por la publicación original de Bethesda) ${ }^{22}$

\begin{tabular}{|lcc|}
\hline Categoría & $\begin{array}{c}\text { \% de nódulos cuya } \\
\text { PAAF corresponde } \\
\text { a cada una de las } \\
\text { categorías }\end{array}$ & $\begin{array}{c}\text { Riesgo } \\
\text { estimado de } \\
\text { malignidad } \\
\text { por categoría }\end{array}$ \\
\hline BETHESDA I & $5-15 \%$ & $1-4 \%$ \\
\hline BETHESDA II & $55-74 \%$ & $0-3 \%$ \\
\hline BETHESDA III & $2-18 \%$ & $5-15 \%$ \\
\hline BETHESDA IV & $2-25 \%$ & $15-30 \%$ \\
\hline BETHESDA V & $1-6 \%$ & $60-75 \%$ \\
\hline BETHESDA VI & $2-5 \%$ & $97-99 \%$ \\
\hline
\end{tabular}


b) Nódulo tiroideo $<1 \mathrm{~cm}$ con criterios ecográficos de alta sospecha, asociado a adenopatías sospechosas, proximidad a tráquea o nervio laríngeo recurrente o sospecha de extensión extratiroidea. En estos casos el especialista asumirá la decisión de estudio con PAAF o seguimiento (Recomendación, GRADE 1).

c) TSH baja o suprimida.

d) Nódulo tiroideo con captación incidental en un 18-FDG PET.

e) Antecedente de irradiación de cuello en la infancia o adolescencia (radioterapia).

f) Nódulo tiroideo en el contexto de los siguientes síndromes: poliposis familiar colon, síndrome de Gardner, síndrome de Turcot, enfermedad de Cowden, complejo de Carney, neoplasia endocrina múltiple tipo-2 (NEM-2), síndrome de Werner.

\subsection{Casos que requieren una derivación urgente} a especialidad:

a) Nódulos tiroideos indoloros con crecimiento rápido en pocas semanas.

b) Nódulos con presencia de adenopatías sospechosas regionales o sospecha de metástasis a distancia.

c) Nódulos sólidos mayores de $4 \mathrm{~cm}$ y/o con síntomas de compresión local atribuibles al nódulo (disfagia, sensación de atoro, disfonía).

\subsection{Casos que requieren derivación a Servicio de} Urgencia: (inmediata)

a) Nódulo tiroideo o bocio multinodular que produzca síntomas compresivos agudos: disnea, estridor laríngeo o disfagia severa.

\subsection{Pacientes que deben ser controlados $y$} observados en atención primaria:

a) Nódulos $<1 \mathrm{~cm}$ sin elementos de sospecha.

b) Nódulos $<1,5 \mathrm{~cm}$ de baja sospecha.

c) Nódulos $<2 \mathrm{~cm}$ de muy baja sospecha.

d) Nódulos $<5 \mathrm{~mm}$ de alta sospecha, sin metástasis ganglionares, sospecha de extensión extratiroidea, adherido a tráquea ni a nervio laríngeo recurrente (Recomendación, GRADE 1).

\subsection{Casos que pueden ser controlados en atención primaria o bien derivados a endocrinología}

a) Nódulos entre 5-10 mm, de alta sospecha, sin signos de extensión extratiroidea ni adenopatías sospechosas (Sugerencia, GRADE 2).

\section{0. ¿Cuál es la conducta según el informe citológico?}

La conducta posterior a la PAAF será determinada por el especialista, incluyendo observación, repetición de la PAAF, eventualmente utilización de estudios moleculares o cirugía según los elementos clínicos, ecográficos e informe citológico $(\text { Anexo } 3)^{15}$.

\section{1. ¿Cómo se realiza el seguimiento de los nódulos que no cumplen criterios para ser puncionados?}

El seguimiento depende de las características ecotomográficas iniciales y de los factores de riesgo del paciente.

En pacientes con nódulos predominantemente quísticos o con características espongiformes, sin factores de riesgo personal o familiar, se sugiere controlar en 1 año con niveles hormonales tiroideos y ecotomografía tiroidea para evaluar crecimiento ( $>50 \%$ del volumen) o aparición de cambios sospechosos de neoplasia. En este último caso se sugiere realizar una PAAF. En caso de nódulo estable, se puede controlar en 12-24 meses con ecotomografía, y posteriormente se puede mantener en seguimiento clínico si ecográficamente estable (Recomendación, GRADE 1) ${ }^{15}$.

En el caso de los nódulos con características ecográficas sospechosas $<1 \mathrm{~cm}$ que no fueron puncionados, se debe controlar con ecografía a los 6 meses y en caso de no haber cambios se debe mantener en control una vez al año con pruebas de función tiroidea y ecografía de manera indefinida ${ }^{15}$.

\section{2. ¿Existe un rol del tratamiento con levotiroxi- na en el manejo de los nódulos tiroideos?}

No se recomienda la terapia supresora (TSH $<0,1 \mathrm{mUI} / \mathrm{L}$ ) con levotiroxina en nódulos tiroideos benignos en áreas con suficiencia de yodo como es Chile, dado que los potenciales efectos adversos sobrepasan el modesto beneficio en la mayoría de los pacientes. El hipertiroidismo iatrogénico se ha asociado a aumento del riesgo de osteoporosis y fracturas en mujeres postmenopáusicas y de arritmias como fibrilación auricular en adultos mayores. No existen datos suficientes y de alta calidad que evalúen la terapia con levotiroxina a dosis no supresoras para prevención del crecimiento de nódulos tiroideos. 


\section{3. ¿Cómo se realiza el seguimiento de los} nódulos puncionados y con resultado benigno?

Debe mantenerse control inicialmente en un año con ecografía y función tiroidea. Si no existe crecimiento, los controles sucesivos dependerán del tamaño del nódulo y características ecográficas (cada 1-3 años), y posteriormente más diferidos según evolución. Deben ser seguidos en atención primaria, salvo aquellos nódulos con PAAF benigna, pero de alto riesgo ecográfico.

\section{4. ¿Qué información se debe adjuntar al momento de derivar a un paciente al especialista?}

Toda derivación a especialista debe contener información mínima con la finalidad de dar una correcta priorización de consulta a especialista (Anexo 4).

\section{Conclusiones del Consenso}

1. ¿A quién y cómo se debe realizar tamizaje de nódulo tiroideo?

- No se recomienda el tamizaje a la población general.

- Se recomienda realizar tamizaje con ecotomografía tiroidea en aquellas poblaciones en las cuales hay un mayor riesgo de cáncer de tiroides (ver texto).

- Se sugiere no realizar ecografía tiroidea de rutina en pacientes con Tiroiditis de Hashimoto, salvo en pacientes con bocio o palpación anormal.

2. ¿Cuáles son las características clínicas a considerar en pacientes que consultan por un nódulo tiroideo?

- Ver texto.

3. ¿Cuáles son los exámenes de laboratorio necesarios para evaluar un paciente con nódulo tiroideo?

- Siempre se debe medir TSH. Si está bajo el rango de normalidad, debiera realizarse un cintigrama tiroideo si el nódulo tiroideo es $>$ de $1 \mathrm{~cm}$.

- No se recomienda medir calcitonina de manera rutinaria (ver texto).

4. ¿Qué estudio imagenológico se debe realizar en un paciente con nódulo tiroideo?
- Se debe realizar una ecotomografía de tiroides en todo paciente en el cual se detecta o se sospecha la presencia de un nódulo tiroideo.

4a.¿Cuáles son los requisitos que debe tener el informe de la ecotomografía?

El informe de la ecografía tiroidea debe incluir:

- Descripción general de la glándula tiroides.

- Descripción de nódulos.

- Descripción de los linfonodos.

- Conclusión: Riesgo de malignidad del nódulo. No debe incluir indicación de punción aspirativa con aguja fina (PAAF), principalmente en aquellos nódulos $<$ a $1 \mathrm{~cm}$.

4b.¿Cuándo realizar Cintigrama tiroideo?

- Deberá solicitarse ante la presencia de nódulo $>1 \mathrm{~cm}$ asociado a TSH bajo el rango normal.

5. ¿Cuáles son las indicaciones de PAAF de un nódulo tiroideo?

Alta sospecha o TIRADS 4b/4c/5:

- Realizar PAAF siempre cuando son $\geq 1 \mathrm{~cm}$.

- Entre 5 y $10 \mathrm{~mm}$, evaluar caso a caso necesidad de PAAF.

- $<5 \mathrm{~mm}$, no puncionar, salvo presencia de elementos de riesgo (ver texto).

Sospecha intermedia o TIRADS 4/4a

- Realizar PAAF siempre cuando son $\geq 1 \mathrm{~cm}$.

Baja sospecha o TIRADS 3

- Realizar PAAF siempre cuando son $\geq 1,5 \mathrm{~cm}$.

Muy baja sospecha o TIRADS 2

- Realizar PAAF cuando son $\geq 2 \mathrm{~cm}$ o también se podría solo observar.

Patrón ecográfico benigna según ATA o TIRADS 2

- No requiere PAAF.

Puncionar siempre ante la presencia de:

- Adenopatías sospechosas.

- Extensión extratiroidea.

- Compromiso traqueal.

- Compromiso de nervio laríngeo recurrente.

6. ¿Cuál es la conducta en nódulos sospechosos menores a $1 \mathrm{~cm}$ ?

- Nódulo sospechoso < $5 \mathrm{~mm}$ : Se recomienda observar (salvo los raros casos que presenten 
ganglios con sospecha de metástasis, extensión extratiroidea, compromiso traqueal o de nervio laríngeo recurrente).

- Nódulo sospechoso entre 5-10 mm: se deben considerar una serie de factores para evaluar la necesidad y posible beneficio de PAAF e intervención en caso de ser un microcarcinoma (ver texto).

7. ¿Cómo es la técnica de PAAF por aguja fina?

- Ver texto.

8. ¿Cómo debe ser analizada la muestra obtenida por PAAF con aguja fina?

- Debe ser hecho por un patólogo con experiencia en citología tiroidea.

- Debe ser reportada usando las categorías Bethesda.

9. ¿Cuáles son las indicaciones de derivación de pacientes con nódulo tiroideo desde Atención Primaria?

9.1. Casos que requieren evaluación en policlínico de endocrinología

- Nódulo tiroideo con criterios ecográficos de sospecha de malignidad $\mathrm{y} \geq 1 \mathrm{~cm}$ con indicación de PAAF (ver indicaciones de PAAF-de nódulo tiroideo).

- Nódulo tiroideo $<1 \mathrm{~cm}$ con criterios ecográficos de alta sospecha (ver texto). En estos casos el especialista asumirá la decisión de estudio con PAAF o seguimiento.

- TSH baja o suprimida.

- Nódulo tiroideo con captación incidental en un 18-FDG PET.

- Antecedente de radioterapia de cuello en la infancia o adolescencia.

- Nódulo tiroideo en el contexto de síndromes genéticos (ver texto).

9.2. Casos que requieren una derivación urgente a especialidad

- Nódulos tiroideos indoloros con crecimiento rápido en pocas semanas.

- Nódulos con presencia de adenopatías sospechosas regionales o sospecha de metástasis a distancia.

- Nódulos sólidos mayores de $4 \mathrm{~cm}$ y/o con síntomas de compresión local atribuibles al nódulo (disfagia, sensación de atoro, disfonía).
9.3. Casos que requieren derivación a Servicio de Urgencia: (inmediata)

- Nódulo tiroideo o bocio multinodular que produzca síntomas compresivos agudos: disnea, estridor laríngeo o disfagia severa.

9.4. Pacientes que deben ser controlados y observados en atención primaria

- Nódulos $<1 \mathrm{~cm}$ sin elementos de sospecha.

- Nódulos $<1,5 \mathrm{~cm}$ de baja sospecha.

- Nódulos $<2 \mathrm{~cm}$ de muy baja sospecha.

- Nódulos $<5 \mathrm{~mm}$ de alta sospecha, sin ninguno de los siguientes elementos: metástasis ganglionares, sospecha de extensión extratiroidea, adherido a tráquea ni a nervio laríngeo recurrente.

9.5. Casos que pueden ser controlados en atención primaria o bien derivados a endocrinología:

- Nódulos entre 5-10 mm, de alta sospecha, sin signos de extensión extratiroidea ni adenopatías sospechosas.

10. ¿Cuál es la conducta según el informe citológico?

- La conducta posterior a la PAAF será determinada por el especialista, incluyendo observación, repetición de la PAAF, eventualmente utilización de estudios moleculares o cirugía según los elementos clínicos, ecográficos e informe citológico (ver texto).

11. ¿Cómo se realiza el seguimiento de los nódulos que no cumplen criterios para ser puncionados?

- El seguimiento depende de las características ecotomográficas iniciales y de los factores de riesgo del paciente (ver texto).

12. ¿Existe un rol del tratamiento con levotiroxina en el manejo de los nódulos tiroideos?

- No se recomienda la terapia supresora con levotiroxina en nódulos tiroideos benignos.

13. ¿Cómo se realiza el seguimiento de los nódulos puncionados y con resultado benigno?

- Debe mantenerse control inicialmente en un año con ecografía y función tiroidea. Deben ser seguidos en atención primaria, salvo aquellos nódulos con PAAF benigna, pero de alto riesgo ecográfico. 


\section{4. ¿Que información se debe adjuntar al momen- to de derivar a un paciente al especialista?}

- Toda derivación a especialista debe contener información mínima con la finalidad de dar una correcta priorización de consulta a especialista (ver texto).

Anexos en archivo suplementario de la Revista Médica de Chile en: http://www.scielo.cl

\section{Referencias}

1. Mazzaferri EL. Management of a solitary thyroid nodule. N Engl J Med 1993; 328 (8): 553-9.

2. Dean DS, Gharib H. Epidemiology of thyroid nodules. Best Pract Res Clin Endocrinol Metab 2008; 22 (6): 90111 .

3. Guth S, Theune U, Aberle J, Galach A, Bamberger CM. Very high prevalence of thyroid nodules detected by high frequency $(13 \mathrm{MHz})$ ultrasound examination. Eur J Clin Invest 2009; 39 (8): 699-706.

4. Mazzaferri EL. Thyroid cancer in thyroid nodules: finding a needle in the haystack. Am J Med 1992; 93 (4): 359-62.

5. Burman KD, Wartofsky L. Thyroid Nodules. N Engl J Med 2015; 373 (24): 2347-56.

6. Hasson F, Keeney S, McKenna H. Research guidelines for the Delphi survey technique. J Adv Nurs 2000; 32: 1008-101.

7. Iglesias ML, Schmidt A, Ghuzlan AA, Lacroix L, Vathaire F, Chevillard S, et al. Radiation exposure and thyroid cancer: a review. Arch Endocrinol Metab 2017; 61 (2): 180-7.

8. Wells SA Jr, Pacini F, Robinson BG, Santoro M. Multiple endocrine neoplasia type 2 and familial medullary thyroid carcinoma: an update. J Clin Endocrinol Metab 2013; 98 (8): 3149-64.

9. Feng X, Milas M, O’Malley M, LaGuardia L, Berber $\mathrm{E}$, Jin J, et al. Characteristics of benign and malignant thyroid disease in familial adenomatous polyposis patients and recommendations for disease surveillance. Thyroid 2015; 25 (3): 325-32.

10. Correa R, Salpea P, Stratakis CA. Carney complex: an update. Eur J Endocrinol 2015; 173 (4): 85-97.

11. Hall JE, Abdollahian DJ, Sinard RJ. Thyroid disease associated with Cowden syndrome: A meta-analysis. Head Neck 2013; 35 (8): 1189-94.
12. Lauper JM, Krause A, Vaughan TL, Monnat RJ Jr. Spectrum and risk of neoplasia in Werner syndrome: a systematic review. PLoS One 2013; 8 (4): e59709.

13. Daumerie C, Maiter D, Gruson D. Serum calcitonin estimation in medullary thyroid cancer: basal or stimulated levels? Thyroid Res 2013; 6 Suppl 1: S4.

14. Soelberg KK, Bonnema SJ, Brix TH, Hegedüs L. Risk of malignancy in thyroid incidentalomas detected by 18F-fluorodeoxyglucose positron emission tomography: a systematic review. Thyroid 2012; 22 (9): 918-25.

15. Haugen BR, Alexander EK, Bible KC, Doherty GM, Mandel SJ, Nikiforov YE, et al. 2015 American Thyroid Association Management Guidelines for Adult Patients with Thyroid Nodules and Differentiated Thyroid Cancer: The American Thyroid Association Guidelines Task Force on Thyroid Nodules and Differentiated Thyroid Cancer. Thyroid 2016; 26 (1): 1-133.

16. Horvath E, Majlis S, Rossi R, Franco C, Niedmann JP, Castro A, et al. An ultrasonogram reporting system for thyroid nodules stratifying cancer risk for clinical management. J Clin Endocrinol Metab 2009; 94 (5): 1748-51.

17. Horvath E, Silva CF, Majlis S, Rodríguez I, Skoknic V, Castro A, et al. Prospective validation of the ultrasound based TIRADS (Thyroid Imaging Reporting And Data System) classification: results in surgically resected thyroid nodules. Eur Radiol 2017; 27 (6): 2619-28.

18. Leboulleux S, Tuttle RM, Pacini F, Schlumberger M. Papillary thyroid microcarcinoma: time to shift from surgery to active surveillance? Lancet Diabetes Endocrinol 2016; 4 (11): 933-42.

19. Ito Y, Miyauchi A, Kihara M, Higashiyama T, Kobayashi K, Miya A. Patient Age Is Significantly Related to the Progression of Papillary Microcarcinoma of the Thyroid Under Observation. Thyroid 2014; 24 (1): 27-34.

20. Ito Y, Miyauchi A, Oda H. Low-risk papillary microcarcinoma of the thyroid: A review of active surveillance trials. Eur J Surg Oncol 2017. http://dx.doi. org/10.1016/j.ejso.2017.03.004 (Epub ahead of print).

21. Miyauchi A. Clinical Trials of Active Surveillance of Papillary Microcarcinoma of the Thyroid. World J Surg 2016; 40 (3): 516-22.

22. Cibas E, Ali S. The Bethesda System for Reporting Thyroid Cytopathology. Thyroid 2009; 19 (11): 1159-65.

23. Pusztaszeria M, Rossi ED, Auger M, Baloch Z, Bishop J, Bongiovanni M, et al. The Bethesda System for Reporting Thyroid Cytopathology: Proposed Modifications and Updates for the Second Edition from an International Panel. Acta Cytologica 2016; 60 (5): 399-405. 